\title{
Some Measures of Picture Fuzzy Sets and Their Application in Multi-attribute Decision Making
}

\author{
Nguyen Van Dinh ${ }^{a}$, Nguyen Xuan Thao ${ }^{a}$ \\ ${ }^{a}$ Department of applied Math-Informatics, Faculty of Information Technology. Vietnam National University of \\ Agriculture (VNUA). Hanoi, Vietnam
}

Received: 26 September 2017; Accepted: 20 March 2018; Published: 08 July 2018

\begin{abstract}
To measure the difference of two fuzzy sets / intuitionistic sets, we can use the distance measure and dissimilarity measure between fuzzy sets. Characterization of distance/dissimilarity measure between fuzzy sets/intuitionistic fuzzy set is important as it has application in different areas: pattern recognition, image segmentation, and decision making. Picture fuzzy set (PFS) is a generalization of fuzzy set and intuitionistic set, so that it have many application. In this paper, we introduce concepts: difference between PFS-sets, distance measure and dissimilarity measure between picture fuzzy sets, and also provide the formulas for determining these values. We also present an application of dissimilarity measures in multi-attribute decision making.
\end{abstract}

Index Terms: Picture fuzzy set (PFS), difference between PFS-sets, distance measure and dissimilarity measure between picture fuzzy sets, multi-attribute decision making.

(C) 2018 Published by MECS Publisher. Selection and/or peer review under responsibility of the Research Association of Modern Education and Computer Science

\section{Introduction}

In many practical problems, we need to compare two objects. Therefore, the question of the process and the way to compare those objects is important. There are some models to measure difference between objects, as a general axiomatic framework for the comparison of fuzzy set. (Bouchon et al. [1]). Fuzzy set and intuitionistic fuzzy set have been used a lot in practical math problems $[6,8,9,11]$. Distance measure between fuzzy sets and intuitionistic fuzzy sets is also important for many practical applications (Ejegwa et al. [4], Hatzimichailidis et al. [6], Lindblad et al. [8], Muthukumar et al. [12]). Besides, dissimilarity measure between fuzzy sets/intuitionistic fuzzy set is also studied and applied in various matters (Li [7], Faghihi [5], Nguyen [13],

* Corresponding author.

E-mail address: nvdinh@vnua.edu.vn,nxthao@vnua.edu.vn 
Mahmood [10]).

In 2014, Cuong introduced the concept of the picture fuzzy set (PFS-sets) [2], in which a given set is represented by three memberships: a degree of positive membership, a degree of negative membership, and a degree of neutral membership. After that, Son gave the applications of the picture fuzzy set in clustering problems in [15, 16, 17]. Nguyen et al. [14] use picture fuzzy sets to applied for Geographic Data Clustering. Van Dinh et al. [18] introduce the picture fuzzy set database. Cuong and Hai [3] studied some fuzzy logic operators for picture fuzzy sets. Nguyen et all [15] investigate the equivalence of two picture fuzzy set and apply them in clustering. But, difference between PFS-sets and dissimilarity between picture fuzzy sets (the concepts are important in application of picture fuzzy sets) are not yet been research.

In this paper, we introduce the concept of difference between PFS-sets, distance measure operators and dissimilarity measure operators between picture fuzzy sets. The rest of paper, in section 2, we recall the concept of picture fuzzy set and we introduce the new concept difference between PFS-sets. The function of distance measure between PFS-sets is defined in section 3. After that, we introduce the function of dissimilarity measure between PFS-sets in section 4. We also illustrate with numerical examples the above measures in decision making in section 5 . In section 6, we apply the dissimilarity measure in the multiple-attribute decision making.

\section{Basic Notions}

\section{Definition 1.}

A picture fuzzy set (PFS) is defined by:

$$
A=\left\{\left(u, \mu_{A}(u), \eta_{A}(u), \gamma_{A}(u)\right) \mid u \in U\right\}
$$

where: $\mu_{A}$ is a positive membership function, $\eta_{A}$ is neural membership function, $\gamma_{A}$ is negative membership function of A, in there: $\mu_{A}(u), \eta_{A}(u), \gamma_{A}(u) \in[0,1]$ and

$$
0 \leq \mu_{A}(u)+\eta_{A}(u)+\gamma_{A}(u) \leq 1 \text {, for all } u \in U \text {. }
$$

- We denote $P F S(U)$ is a collection of picture fuzzy set on $U$. In which:

$$
U=\{(u, 1,0,0) \mid u \in U\}
$$

and:

$$
\varnothing=\{(u, 0,0,1) \mid u \in U\} .
$$

- For $A, B \in P F S(U)$, then:

+ Union of $A$ and $B$ is defined by:

$$
A \cup B=\left\{\left(u, \mu_{A \cup B}(u), \eta_{A \cup B}(u), \gamma_{A \cup B}(u)\right) \mid u \in U\right\}
$$

where:

$$
\begin{aligned}
& \mu_{\mathrm{A} \cup \mathrm{B}}(\mathrm{u})=\max \left\{\mu_{\mathrm{A}}(\mathrm{u}), \mu_{\mathrm{B}}(\mathrm{u})\right\}, \\
& \eta_{\mathrm{A} \cup \mathrm{B}}(\mathrm{u})=\min \left\{\eta_{\mathrm{A}}(\mathrm{u}), \eta_{\mathrm{B}}(\mathrm{u})\right\},
\end{aligned}
$$




$$
\gamma_{\mathrm{A} \cup B}(\mathrm{u})=\min \left\{\gamma_{\mathrm{A}}(\mathrm{u}), \gamma_{\mathrm{B}}(\mathrm{u})\right\}
$$

+ Intersection of $A$ and $B$ is defined by:

$$
A \cap B=\left\{\left(u, \mu_{A \cap B}(u), \eta_{A \cap B}(u), \gamma_{A \cap B}(u)\right) \mid u \in U\right\}
$$

Where:

$$
\begin{aligned}
& \mu_{A \cap B}(u)=\min \left\{\mu_{A}(u), \mu_{B}(u)\right\}, \\
& \eta_{A \cap B}(u)=\min \left\{\eta_{A}(u), \eta_{B}(u)\right\}, \\
& \gamma_{A \cap B}(u)=\max \left\{\gamma_{A}(u), \gamma_{B}(u)\right\} .
\end{aligned}
$$

+ Subset: $A \subset B$ iff $\left\{\begin{array}{l}\mu_{A}(u) \leq \mu_{B}(u) \\ \eta_{A}(u) \leq \eta_{B}(u) . \\ \gamma_{A}(u) \geq \gamma_{B}(u)\end{array}\right.$.

Now, we define an operator called difference between picture fuzzy sets.

\section{Definition 2.}

An operator - : $P F S(U) \times P F S(U) \rightarrow P F S(U)$ is a difference between PFS-sets if it satisfies properties:

(D1) $A \subset B$ iff $A-B=\emptyset$,

(D2) If $B \subset C$ then $B-A \subset C-A$,

(D3) $(A \cap C)-(B \cap C) \subset A-B$,

(D4) $(A \cup C)-(B \cup C) \subset A-B$,

For all $A, B, C \in P F S(U)$.

\section{Theorem 1.}

The function - : $P F S(U) \times P F S(U) \rightarrow P F S(U)$ given by:

$$
A-B=\left\{\left(u, \mu_{A-B}(u), \eta_{A-B}(u), \gamma_{A-B}(u)\right) \mid u \in U\right\},
$$

where:

$$
\begin{array}{r}
\mu_{A-B}(u)=\max \left\{\left(0, \mu_{A}(u)-\mu_{B}(u)\right\},\right. \\
\eta_{A-B}(u)=\max \left\{0, \eta_{A}(u)-\eta_{B}(u)\right\}, \\
\gamma_{A-B}(u)=\left\{\begin{array}{c}
1-\mu_{A-B}(u)-\eta_{A-B}(u) \text { if } \gamma_{A}(u)>\gamma_{B}(u) \\
\min \left\{\begin{array}{c}
1+\gamma_{A}(u)-\gamma_{B}(u), \\
1-\mu_{A-B}(u)-\eta_{A-B}(u)
\end{array}\right\} \text { if } \gamma_{A}(u) \leq \gamma_{B}(u)
\end{array}\right.
\end{array}
$$


is a difference between PFS-sets.

Proof.

It is easy to see that

$$
0 \leq \mu_{A-B}(u)+\eta_{A-B}(u)+\gamma_{A-B}(u) \leq 1
$$

for all $u \in U$.

We verify all condition in definition 2 :

$\circ \quad$ With condition (D1).

$+A \subset B \Rightarrow A-B=\varnothing$ is obvious.

+ If $A-B=\emptyset$ then

$$
\begin{aligned}
& \mu_{A-B}(u)=\max \left(0, \mu_{A}(u)-\mu_{B}(u)\right\}=0, \\
& \eta_{A-B}(u)=\max \left\{0, \eta_{A}(u)-\eta_{B}(u)\right\}=0
\end{aligned}
$$

so that $\mu_{A}(u) \leq \mu_{B}(u)$ and $\eta_{A}(u) \leq \eta_{B}(u)$;

Hence $\gamma_{A-B}(u)=1$ so that $\gamma_{A}(u) \geq \gamma_{B}(u)$.

It means that $A \subset B$.

$\circ$ With condition (D2).

With $B \subset C$, we have

$$
\mu_{B}(u) \leq \mu_{C}(u), \eta_{B}(u) \leq \eta_{C}(u) \text { and } \gamma_{B}(u) \geq \gamma_{A}(u) .
$$

So that:

$$
\begin{gathered}
\mu_{B-A}(u)=\max \left(0, \mu_{B}(u)-\mu_{A}(u)\right\} \\
\leq \max \left(0, \mu_{C}(u)-\mu_{A}(u)\right\} \\
=\mu_{C-A}(u), \\
\eta_{B-A}(u)=\max \left(0, \eta_{B}(u)-\eta_{A}(u)\right\} \\
\leq \max \left(0, \eta_{C}(u)-\eta_{A}(u)\right\} \\
=\eta_{C-A}(u) .
\end{gathered}
$$

With negative membership function, we consider some cases:

If $\gamma_{A}(u) \leq \gamma_{C}(u) \leq \gamma_{B}(u)$ then 


$$
\begin{gathered}
\gamma_{B-A}(u)=1-\mu_{B-A}(u)-\eta_{B-A}(u) \\
\geq 1-\mu_{C-A}(u)-\eta_{C-A}(u)=\gamma_{C-A}(u)
\end{gathered}
$$

If $\gamma_{C}(u) \leq \gamma_{A}(u) \leq \gamma_{B}(u)$ then

$$
\begin{gathered}
\gamma_{B-A}(u)=1-\mu_{B-A}(u)-\eta_{B-A}(u) \\
\geq 1-\mu_{C-A}(u)-\eta_{C-A}(u) .
\end{gathered}
$$

So that

$$
\gamma_{B-A}(u) \geq \min \left\{\begin{array}{c}
1+\gamma_{A}(u)-\gamma_{C}(u) \\
1-\mu_{C-A}(u)-\eta_{C-A}(u)
\end{array}\right\}=\gamma_{C-A}(u)
$$

If $\gamma_{C}(u) \leq \gamma_{B}(u) \leq \gamma_{A}(u)$ then

$$
\begin{gathered}
\gamma_{B-A}(u)=1-\mu_{B-A}(u)-\eta_{B-A}(u) \\
\geq 1-\mu_{C-A}(u)-\eta_{C-A}(u)
\end{gathered}
$$

and

$$
\gamma_{B}(u)-\gamma_{A}(u) \geq \gamma_{C}(u)-\gamma_{A}(u)
$$

So that:

$$
\begin{gathered}
\gamma_{B-A}(u)=\min \left\{\begin{array}{c}
1+\gamma_{A}(u)-\gamma_{B}(u), \\
1-\mu_{A-B}(u)-\eta_{A-B}(u)
\end{array}\right\} \\
\geq \min \left\{\begin{array}{c}
1+\gamma_{A}(u)-\gamma_{C}(u), \\
1-\mu_{C-A}(u)-\eta_{C-A}(u)
\end{array}\right\} \\
=\gamma_{C-A}(u) .
\end{gathered}
$$

- Similarity, it is possible to show that conditions (D3) and (D4) are also satisfied.

Example 1. Given $U=\left\{u_{1}, u_{2}, u_{3}\right\}$ and two PFS-sets:

$$
A=\left\{\begin{array}{l}
\left(u_{1}, 0.7,0.2,0.1\right), \\
\left(u_{2}, 0.6,0.1,0.1\right), \\
\left(u_{3}, 0.6,0.1,0.2\right)
\end{array}\right\}, B=\left\{\begin{array}{c}
\left(u_{1}, 0.6,0.3,0.1\right) \\
\left(u_{2}, 0.7,0.05,0.2\right), \\
\left(u_{3}, 0.4,0.4,0.1\right)
\end{array}\right\}
$$

Then, computing by Eq.(1) in theorem 1, we have: 


$$
A-B=\left\{\begin{array}{c}
\left(u_{1}, 0.1,0.0,0.9\right) \\
\left(u_{2}, 0.0,0.05,0.9\right) \\
\left(u_{3}, 0.2,0.0,0.8\right)
\end{array}\right\}
$$

\section{Distance Mesure of Picture Fuzy Sets}

In this section, we define the distance measure between picture fuzzy sets.

\section{Definition 3.}

A function $D: \operatorname{PFS}(U) \times \operatorname{PFS}(U) \rightarrow[0,+\infty)$ is a distance measure between PFS-sets if it satisfies follow properties

(i) PF-dist 1: $D(A, B)=0$ iff $A=B$,

(ii) PF-dist 2: $D(A, B)=D(B, A)$, for all $A, B \in P F S(U)$,

(iii) PF-dist 3: $D(A, C) \leq D(A, B)+D(B, C)$, for all $A, B, C \in P F S(U)$.

There are many formulas that determine the distance between two picture fuzzy sets.

\section{Theorem 2.}

Given $U=\left\{u_{1}, u_{2}, \ldots, u_{n}\right\}$ is an universe set. For $A, B \in P F S(U)$. We have some distance measure between picture fuzzy sets

(i)

$$
D_{H}(A, B)=\frac{1}{3 n} \sum_{i=1}^{n}\left[\begin{array}{c}
\left|\mu_{A}\left(u_{i}\right)-\mu_{B}\left(u_{i}\right)\right| \\
+\left|\eta_{A}\left(u_{i}\right)-\eta_{B}\left(u_{i}\right)\right| \\
+\left|\gamma_{A}\left(u_{i}\right)-\gamma_{B}\left(u_{i}\right)\right|
\end{array}\right]
$$

(ii) $\quad D_{E}(A, B)=\frac{1}{n}\left\{\sum_{i=1}^{n}\left[\begin{array}{c}\left(\mu_{A}\left(u_{i}\right)-\mu_{B}\left(u_{i}\right)\right)^{2} \\ +\left(\eta_{A}\left(u_{i}\right)-\eta_{B}\left(u_{i}\right)\right)^{2} \\ +\left(\gamma_{A}\left(u_{i}\right)-\gamma_{B}\left(u_{i}\right)\right)^{2}\end{array}\right]\right\}^{\frac{1}{2}}$

(iii) $D_{H}^{m}(A, B)=\frac{1}{n} \sum_{i=1}^{n} \max \left[\begin{array}{l}\left|\mu_{A}\left(u_{i}\right)-\mu_{B}\left(u_{i}\right)\right|, \\ \left|\eta_{A}\left(u_{i}\right)-\eta_{B}\left(u_{i}\right)\right|, \\ \left|\gamma_{A}\left(u_{i}\right)-\gamma_{B}\left(u_{i}\right)\right|\end{array}\right]$

(iv) $D_{E}^{m}(A, B)=\frac{1}{n}\left\{\sum_{i=1}^{n} \max \left[\begin{array}{l}\left|\mu_{A}\left(u_{i}\right)-\mu_{B}\left(u_{i}\right)\right|^{2}, \\ \left|\eta_{A}\left(u_{i}\right)-\eta_{B}\left(u_{i}\right)\right|^{2}, \\ \left|\gamma_{A}\left(u_{i}\right)-\gamma_{B}\left(u_{i}\right)\right|^{2}\end{array}\right]\right\}^{\frac{1}{2}}$

We easy to verify that the functions in theorem 2 are satisfies properties of distance measure between picture fuzzy sets (def. 3). In there, $D_{E}(A, B)$ is usually used to measure the distance of objects in geometry, $D_{H}(A, B)$ is used in the information theory.

Example 2. Assume that there are two patterns denoted by picture fuzzy sets on $U=\left\{u_{1}, u_{2}, u_{3}\right\}$ as follows: 


$$
\begin{gathered}
A_{1}=\left\{\begin{array}{l}
\left(u_{1}, 0.609,0.091,0.298\right), \\
\left(u_{2}, 0.651,0.231,0.059\right), \\
\left(u_{3}, 0.792,0.095,0.106\right)
\end{array}\right\}, \\
A_{2}=\left\{\begin{array}{l}
\left(u_{1}, 0.291,0.365,0.134\right), \\
\left(u_{2}, 0.438,0.468,0.065\right), \\
\left(u_{3}, 0.816,0.169,0.006\right)
\end{array}\right\} . \\
A_{3}=\left\{\begin{array}{l}
\left(u_{1}, 0.679,0.215,0.014\right), \\
\left(u_{2}, 0.239,0.617,0.086\right), \\
\left(u_{3}, 0.917,0.045,0.011\right)
\end{array}\right\}
\end{gathered}
$$

Then we have the results:

+ Using the Eq.(2) we obtain:

$$
\begin{aligned}
& D_{H}\left(A_{1}, A_{2}\right)=0.156667 ; \\
& D_{H}\left(A_{1}, A_{3}\right)=0.174778 ; \\
& D_{H}\left(A_{2}, A_{3}\right)=0.139667 ;
\end{aligned}
$$

+ Using the Eq.(3) we have

$$
\begin{aligned}
& D_{E}\left(A_{1}, A_{2}\right)=0.56632 ; \\
& D_{E}\left(A_{1}, A_{3}\right)=0.66899 ; \\
& D_{E}\left(A_{2}, A_{3}\right)=0.52468 .
\end{aligned}
$$

+ By same way with Eq.(4) we achieve

$$
\begin{aligned}
& D_{H}^{m}\left(A_{1}, A_{2}\right)=0.655 ; \\
& D_{H}^{m}\left(A_{1}, A_{3}\right)=0.821 ; \\
& D_{H}^{m}\left(A_{2}, A_{3}\right)=0.711 ;
\end{aligned}
$$

+ Finally, we using the Eq.(5), we have

$$
\begin{gathered}
D_{E}^{m}\left(A_{1}, A_{2}\right)=0.409 ; \\
D_{E}^{m}\left(A_{1}, A_{3}\right)=0.5158 ; \\
D_{E}^{m}\left(A_{2}, A_{3}\right)=0.4533 .
\end{gathered}
$$




\section{Dissimilarity of Picture Fuzy Sets}

In this section, we introduce the concept of dissimilarity for picture fuzzy sets.

\section{Definition 4.}

A function $D M: P F S(U) \times P F S(U) \rightarrow R$ is a dissimilarity measure between PFS-sets if it satisfies follow properties:

(i) PF-Diss 1: $D M(A, B)=D M(B, A)$

(ii) PF-Diss 2: $D M(A, A)=0$.

(iii) PF-Diss 3: If $A \subset B \subset C$ then

$$
D M(A, C) \geq \max \{D M(A, B), D M(B, C)\}
$$

for all $A, B, C \in P F S(U)$.

\section{Theorem 3.}

Given $U=\left\{u_{1}, u_{2}, \ldots, u_{n}\right\}$ is an universe set. For any $A, B \in P F S(U)$, we define a function $D M_{C}(A, B): P F S(U) \times P F S(U) \rightarrow R$ is defined by:

$$
D M_{C}(A, B)=\frac{1}{3 n} \sum_{i=1}^{n}\left[\begin{array}{c}
\left|S_{A}\left(u_{i}\right)-S_{B}\left(u_{i}\right)\right| \\
+\left|\eta_{A}\left(u_{i}\right)-\eta_{B}\left(u_{i}\right)\right|
\end{array}\right]
$$

Where:

$$
\begin{gathered}
S_{A}\left(u_{i}\right)=\left|\mu_{A}\left(u_{i}\right)-\gamma_{A}\left(u_{i}\right)\right|, \text { and } \\
S_{B}\left(u_{i}\right)=\left|\mu_{B}\left(u_{i}\right)-\gamma_{B}\left(u_{i}\right)\right|
\end{gathered}
$$

Then, $D M_{C}(A, B)$ is a dissimilarity measure between PFS-sets.

\section{Proof.}

We check that $D M_{C}$ satisfies the conditions of definition 3. Indeed, we have:

PF-Diss 1 and PF-Diss 2 are obviously.

With PF-Diss 3, if $A \subset B \subset C$ we have

$$
\left\{\begin{array}{l}
\mu_{A}\left(u_{i}\right) \leq \mu_{B}\left(u_{i}\right) \leq \mu_{C}\left(u_{i}\right) \\
\eta_{A}\left(u_{i}\right) \leq \eta_{B}\left(u_{i}\right) \leq \eta_{C}\left(u_{i}\right) \\
\gamma_{A}\left(u_{i}\right) \geq \gamma_{B}\left(u_{i}\right) \geq \gamma_{C}\left(u_{i}\right)
\end{array}\right.
$$

for all $u_{i} \in U$.

So that:

$$
S_{A}\left(u_{i}\right)=\left|\mu_{A}\left(u_{i}\right)-\gamma_{A}\left(u_{i}\right)\right|
$$




$$
\begin{gathered}
\geq S_{B}\left(u_{i}\right)=\left|\mu_{B}\left(u_{i}\right)-\gamma_{B}\left(u_{i}\right)\right|, \\
S_{B}\left(u_{i}\right)=\left|\mu_{B}\left(u_{i}\right)-\gamma_{B}\left(u_{i}\right)\right| \\
\geq S_{C}\left(u_{i}\right)=\left|\mu_{C}\left(u_{i}\right)-\gamma_{C}\left(u_{i}\right)\right|
\end{gathered}
$$

and

$$
\left|\eta_{A}\left(u_{i}\right)-\eta_{C}\left(u_{i}\right)\right| \geq \max \left\{\left|\eta_{A}\left(u_{i}\right)-\eta_{B}\left(u_{i}\right)\right|,\left|\eta_{B}\left(u_{i}\right)-\eta_{C}\left(u_{i}\right)\right|\right\}
$$

Hence

$$
D M_{C}(A, C) \geq \max \left\{D M_{C}(A, B), D M_{C}(B, C)\right\} .
$$

It means PF-Diss 3 satisfy.

We have some dissimilarity measure in theorem 3 , as follows.

\section{Theorem 4.}

Given $U=\left\{u_{1}, u_{2}, \ldots, u_{n}\right\}$ is an universe set. For any $A, B \in P F S(U)$. We have:

(i) $D M_{H}(A, B)=\frac{1}{3 n} \sum_{i=1}^{n}\left[\begin{array}{c}\left|\mu_{A}\left(u_{i}\right)-\mu_{B}\left(u_{i}\right)\right| \\ +\left|\eta_{A}\left(u_{i}\right)-\eta_{B}\left(u_{i}\right)\right| \\ +\left|\gamma_{A}\left(u_{i}\right)-\gamma_{B}\left(u_{i}\right)\right|\end{array}\right]$

(ii) $D M_{L}(A, B)=\frac{1}{5 n} \sum_{i=1}^{n}\left[\begin{array}{c}\left|S_{A}\left(u_{i}\right)-S_{B}\left(u_{i}\right)\right| \\ +\left|\mu_{A}\left(u_{i}\right)-\mu_{B}\left(u_{i}\right)\right| \\ +\left|\eta_{A}\left(u_{i}\right)-\eta_{B}\left(u_{i}\right)\right| \\ +\left|\gamma_{A}\left(u_{i}\right)-\gamma_{B}\left(u_{i}\right)\right|\end{array}\right]$

(iii) $D M_{O}(A, B)=\frac{1}{\sqrt{3 n}} \sum_{i=1}^{n}\left[\begin{array}{c}\left(\mu_{A}\left(u_{i}\right)-\mu_{B}\left(u_{i}\right)\right)^{2} \\ +\left(\eta_{A}\left(u_{i}\right)-\eta_{B}\left(u_{i}\right)\right)^{2} \\ +\left(\gamma_{A}\left(u_{i}\right)-\gamma_{B}\left(u_{i}\right)\right)^{2}\end{array}\right]^{\frac{1}{2}}$

are the dissimilarity measure between picture fuzzy sets.

The proof of this theorem is similar to the theorem 3.

Example 3. Assume that there are two patterns denoted by picture fuzzy sets on $U=\left\{u_{1}, u_{2}, u_{3}\right\}$ as follows

$$
\begin{gathered}
A_{1}=\left\{\begin{array}{l}
\left(u_{1}, 0.609,0.091,0.298\right), \\
\left(u_{2}, 0.651,0.231,0.059\right), \\
\left(u_{3}, 0.792,0.095,0.106\right)
\end{array}\right\}, \\
A_{2}=\left\{\begin{array}{c}
\left(u_{1}, 0.291,0.365,0.134\right), \\
\left(u_{2}, 0.438,0.468,0.065\right), \\
\left(u_{3}, 0.816,0.169,0.006\right)
\end{array}\right\} .
\end{gathered}
$$




$$
A_{3}=\left\{\begin{array}{l}
\left(u_{1}, 0.679,0.215,0.014\right), \\
\left(u_{2}, 0.239,0.617,0.086\right), \\
\left(u_{3}, 0.917,0.045,0.011\right)
\end{array}\right\}
$$

+ Using Eq.(6) we have:

$$
\begin{aligned}
& D M_{C}\left(A_{1}, A_{2}\right)=0.360667 ; \\
& D M_{C}\left(A_{1}, A_{3}\right)=0.524333 ; \\
& D M_{C}\left(A_{2}, A_{3}\right)=0.415667 ;
\end{aligned}
$$

+ Using Eq.(7) we have

$$
\begin{aligned}
& D M_{H}\left(A_{1}, A_{2}\right)=0.145556 ; \\
& D M_{H}\left(A_{1}, A_{3}\right)=0.139111 ; \\
& D M_{H}\left(A_{2}, A_{3}\right)=0.164222 ;
\end{aligned}
$$

+ Using Eq.(8) we obtain

$$
\begin{gathered}
D M_{L}\left(A_{1}, A_{2}\right)=0.193267 \\
D M_{L}\left(A_{1}, A_{3}\right)=0.1728 \\
D M_{L}\left(A_{2}, A_{3}\right)=0.213467
\end{gathered}
$$

+ Finally with Eq.(9) we get the results:

$$
\begin{aligned}
& D M_{O}\left(A_{1}, A_{2}\right)=0.188774 ; \\
& D M_{O}\left(A_{1}, A_{3}\right)=0.174893 ; \\
& D M_{O}\left(A_{2}, A_{3}\right)=0.222998 ;
\end{aligned}
$$

\section{Numerical Examples for using New Measures in Partern Recognition}

In this section, we will give some examples using distance and dissimilarity measure $D M(A, B)$ in decision making. Note that when using similar measure, there are two patterns $A_{1}, A_{2}$ and a sample $B$. If $D M\left(A_{1}, B\right)<$ $D M\left(A_{2}, B\right)$ the we consider that sample $B$ belongs to the pattern $A_{1}$.

Example 4. Assume that there are two patterns denoted by picture fuzzy sets on $U=\left\{u_{1}, u_{2}, u_{3}\right\}$ as follows

$$
A_{1}=\left\{\begin{array}{c}
\left(u_{1}, 0.1,0.1,0.1\right), \\
\left(u_{2}, 0.1,0.4,0.3\right), \\
\left(u_{3}, 0.1,0.0,0.9\right)
\end{array}\right\}
$$




$$
A_{2}=\left\{\begin{array}{l}
\left(u_{1}, 0.7,0.1,0.2\right), \\
\left(u_{2}, 0.1,0.1,0.8\right), \\
\left(u_{3}, 0.1,0.1,0.7\right)
\end{array}\right\}
$$

Now, there is a sample:

$$
B=\left\{\begin{array}{l}
\left(u_{1}, 0.4,0.0,0.4\right), \\
\left(u_{2}, 0.6,0.1,0.2\right), \\
\left(u_{3}, 0.1,0.1,0.8\right)
\end{array}\right\}
$$

Question: which pattern does B belong to?

+ Applying the distant measure $D_{H}(A, B)$ (i.e. Eq. (2)) we have:

$$
D_{H}\left(A_{1}, B\right)=D_{H}\left(A_{2}, B\right)=0.2
$$

+ Applying the dissimilarity measure $D M_{L}(A, B)$ in Eq.(8) we have:

$$
D M_{L}\left(A_{1}, B\right)=\frac{2.1}{15}<D M_{L}\left(A_{2}, B\right)=\frac{2.7}{15} .
$$

In this example, we see that using the distant measure $D_{H}(A, B)$ can not be used to classify the sample $B$. But, we can see that $B$ belongs to pattern $A_{1}$ if we use the dissimilarity measure $D M_{L}(A, B)$.

Example 5. Assume that there are three patterns denoted by picture fuzzy sets on $U=\left\{u_{1}, u_{2}, u_{3}\right\}$ as follows

$$
\begin{gathered}
A_{1}=\left\{\begin{array}{l}
\left(u_{1}, 0.4,0.5,0.1\right), \\
\left(u_{2}, 0.7,0.1,0.1\right), \\
\left(u_{3}, 0.3,0.3,0.2\right)
\end{array}\right\}, \\
A_{2}=\left\{\begin{array}{l}
\left(u_{1}, 0.5,0.4,0.0\right), \\
\left(u_{2}, 0.7,0.2,0.1\right), \\
\left(u_{3}, 0.4,0.3,0.2\right)
\end{array}\right\} . \\
A_{3}=\left\{\begin{array}{l}
\left(u_{1}, 0.4,0.4,0.1\right), \\
\left(u_{2}, 0.6,0.1,0.1\right), \\
\left(u_{3}, 0.4,0.1,0.4\right)
\end{array}\right\}
\end{gathered}
$$

Now, there is a sample:

$$
B=\left\{\begin{array}{l}
\left(u_{1}, 0.1,0.1,0.6\right), \\
\left(u_{2}, 0.7,0.1,0.2\right), \\
\left(u_{3}, 0.8,0.1,0.1\right)
\end{array}\right\}
$$

Question: which pattern does B belong to?

+ Applying the dissimilarity measure $D M_{L}(A, B)$ in Eq.(8) we have: 


$$
\begin{gathered}
D M_{L}\left(A_{1}, B\right)=D M_{L}\left(A_{3}, B\right)=\frac{2.1}{9} ; \\
D M_{L}\left(A_{2}, B\right)=\frac{2.2}{9} .
\end{gathered}
$$

+ Applying the distance measure $D_{E}(A, B)$ in Eq.(3) we have:

$$
\begin{gathered}
D_{E}\left(A_{1}, B\right)=0.9 ; \\
D_{E}\left(A_{2}, B\right)=0.916515139 ; \\
D_{E}\left(A_{3}, B\right)=0.8366600265
\end{gathered}
$$

In this example, we see that using the dissimilarity measure $D M_{H}(A, B)$ can not be used to classify the sample $B$. But, we can see that $B$ belongs to pattern $A_{3}$ if we use the distance measure $D_{E}(A, B)$.

Example 6. Assume that there are three patterns denoted by picture fuzzy sets on $U=\left\{u_{1}, u_{2}, u_{3}, u_{4}\right\}$ as follows:

$$
\begin{aligned}
& A_{1}=\left\{\begin{array}{l}
\left(u_{1}, 0.3,0.4,0.1\right),\left(u_{2}, 0.3,0.4,0.1\right), \\
\left(u_{3}, 0.6,0.1,0.2\right),\left(u_{4}, 0.6,0.1,0.2\right)
\end{array}\right\} \\
& A_{2}=\left\{\begin{array}{l}
\left(u_{1}, 0.4,0.4,0.1\right),\left(u_{2}, 0.3,0.2,0.4\right), \\
\left(u_{3}, 0.6,0.1,0.3\right),\left(u_{4}, 0.5,0.2,0.2\right)
\end{array}\right\} \\
& A_{3}=\left\{\begin{array}{c}
\left(u_{1}, 0.4,0.4,0.1\right),\left(u_{2}, 0.3,0.1,0.3\right), \\
\left(u_{3}, 0.6,0.1,0.2\right),\left(u_{4}, 0.5,0.2,0.1\right)
\end{array}\right\}
\end{aligned}
$$

Now, there is a sample:

$$
B=\left\{\begin{array}{c}
\left(u_{1}, 0.35,0.65,0\right),\left(u_{2}, 0.55,0.35,0.1\right) \\
\left(u_{3}, 0.65,0.1,0.1\right),\left(u_{4}, 0.6,0.15,0.2\right)
\end{array}\right\}
$$

Question: which pattern does B belong to?

+ Applying the distance measure $D_{H}^{m}(A, B)$ in Eq.(4) we have:

$$
\begin{gathered}
D_{H}^{m}\left(A_{1}, B\right)=D_{H}^{m}\left(A_{3}, B\right)=0.7 ; \\
D_{H}^{m}\left(A_{2}, B\right)=0.85 .
\end{gathered}
$$

+ Applying the dissimilarity measure $D M_{C}(A, B)$ in Eq.(6) we have:

$$
\begin{gathered}
D M_{C}\left(A_{1}, B\right)=0.0875 ; \\
D M_{C}\left(A_{2}, B\right)=D M_{C}\left(A_{3}, B\right)=0.1
\end{gathered}
$$


In this example, we see that using the distance measure $D_{H}^{m}(A, B)$ can not be used to classify the sample $B$. But, we can see that $B$ belongs to pattern $A_{1}$ if we use the dissimilarity measure $D M_{C}(A, B)$.

Example 7. Assume that there are two patterns denoted by picture fuzzy sets on $U=\left\{u_{1}, u_{2}\right\}$ as follows

$$
\begin{aligned}
& A_{1}=\left\{\begin{array}{c}
\left(u_{1}, 0.4,0.5,0.1\right), \\
\left(u_{2}, 0.3,0.4,0.2\right)
\end{array}\right\}, \\
& A_{2}=\left\{\begin{array}{c}
\left(u_{1}, 0.5,0.4,0.1\right), \\
\left(u_{2}, 0.4,0.3,0.1\right)
\end{array}\right\} .
\end{aligned}
$$

Now, there is a sample:

$$
B=\left\{\begin{array}{l}
\left(u_{1}, 0.1,0.1,0.1\right) \\
\left(u_{2}, 0.5,0.5,0.0\right)
\end{array}\right\}
$$

Question: which pattern does B belong to?

+ Applying the distant measure $D_{E}^{m}(A, B)$ (i.e. Eq.(5)) we have:

$$
D_{E}^{m}\left(A_{1}, B\right)=D_{E}^{m}\left(A_{2}, B\right)=0.44721
$$

+ Applying the dissimilarity measure $D M_{O}(A, B)$ (i.e. Eq.(9)) we have:

$$
\begin{gathered}
D M_{O}\left(A_{1}, B\right)=0.3265 ; \\
D M_{O}\left(A_{2}, B\right)=0.3041241 .
\end{gathered}
$$

In this example, we see that using the distant measure $D_{E}^{m}(A, B)$ can not be used to classify the sample $B$. But, we can see that $B$ belongs to pattern $A_{2}$ if we use the dissimilarity measure $D M_{O}(A, B)$.

\section{Appliction in Multiple Attribute Decision Making}

In modern decision science, the multiple attribute decision making is an important research and has been widely in many fields, such as economy, management, medical and so on. In this section, we will use our dissimilarity measure to apply in a multiple attribute decision making. It present in Example 6.

Example 8. This example is developed from the example that presented by $\mathrm{Xu}$ (in 2007). This example is to evaluate the university faculty for tenure and promotion. There are six faculty candidates (alternatives), $A_{i},(i=1,2, \ldots, 6)$, to be evaluated, the used attributes are $G_{1}$ : teaching, $G_{2}$ : research and $G_{3}$ : service. In which, the weights of each attribute $G_{j}$ is $w_{j}, j=1,2,3 ; \sum_{j=1}^{3} w_{j}=1$ the used weight vector is $w=(0.55,0.25,0.2)$.

The picture fuzzy decision matrix $R=\left(r_{i j}\right)_{6 \times 3}$, where $r_{i j}=\left(\mu_{i j}, \eta_{i j}, \gamma_{i j}\right),(i=1,2, \ldots, 6 ; j=1,2,3)$ is shown in Table 1.

We denote the alternatives $A_{i},(i=1,2, \ldots, 6)$ and $\left.A_{b}(j)=(1,0,0) ; j=1,2,3\right)$. Then, we have $A_{b}=$ $\left(A_{b}(1), A_{b}(2), A_{b}(3)\right)$ be the largest picture fuzzy number. 
Table 1. Picture Fuzzy Decision Matrix R

\begin{tabular}{|c|c|c|c|}
\hline & $\mathrm{G}_{1}$ & $G_{2}$ & $G_{3}$ \\
\hline $\mathrm{A}_{1}$ & $(0.61,0.08,0.11)$ & $(0.59,0.04,0.26)$ & $(0.41,0.36,0.08)$ \\
\hline $\mathrm{A}_{2}$ & $(0.54,0.45,0.01)$ & $(0.5,0.15,0.25)$ & $(0.66,0.12,0.01)$ \\
\hline $\mathrm{A}_{3}$ & $(0.72,0.23,0.03)$ & $(0.53,0.05,0.35)$ & $(0.5,0.03,0.08)$ \\
\hline $\mathrm{A}_{4}$ & $(0.63,0.3,0.06)$ & $(0.59,0.29,0.08)$ & $(0.58,0.07,0.16)$ \\
\hline $\mathrm{A}_{5}$ & $(0.59,0.3,0.09)$ & $(0.41,0.57,0.01)$ & $(0.59,0.19,0.1)$ \\
\hline $\mathrm{A}_{6}$ & $(0.73,0.1,0.15)$ & $(0.54,0.37,0.06)$ & $(0.46,0.24,0.06)$ \\
\hline
\end{tabular}

The dissimilarity measure between alternatives $A_{i}$ and the possible ideal alternative $A_{b}$ is defined as follows:

$$
\operatorname{DM}\left(A_{i}, A_{b}\right)=\sum_{j=1}^{3} w_{j} D M\left(r_{i j}, A_{b}(j)\right)
$$

where $D M\left(r_{i j}, A_{b}(j)\right)$ is the dissimilarity measure of $r_{i j}$ and $A_{b}(j), j=1,2,3 ; i=1,2, \ldots, 6$.

So that, the smaller of the dissimilarity measure is the better alternatives.

We consider the calculated results according to the measurements in the section 4 .

+ Apply the Eq.(10), with the dissimilarity measure $D M_{H}(A, B)$ in the Eq.(7) we have:

$$
\begin{gathered}
D M_{H}\left(A_{1}, A_{b}\right)=0.23417 \\
D M_{H}\left(A_{2}, A_{b}\right)=0.275 \\
D M_{H}\left(A_{3}, A_{b}\right)=0.21217 \\
D M_{H}\left(A_{4}, A_{b}\right)=0.24217 \\
D M_{H}\left(A_{5}, A_{b}\right)=0.29083 \\
D M_{H}\left(A_{6}, A_{b}\right)=0.2255
\end{gathered}
$$

Here, we have ranking:

$$
A_{3}>A_{6}>A_{1}>A_{4}>A_{2}>A_{5},
$$

where the symbol " > " mean that order relation "superior". Hence, the most faculty (alternative) is $A_{3}$.

+ Apply the Eq.(10), with the dissimilarity measure $D M_{O}(A, B)$ in the Eq.(9) we have:

$$
\begin{gathered}
D M_{O}\left(A_{1}, A_{b}\right)=0.1627 \\
D M_{O}\left(A_{2}, A_{b}\right)=0.19027 \\
D M_{O}\left(A_{3}, A_{b}\right)=0.14949
\end{gathered}
$$




$$
\begin{aligned}
& D M_{O}\left(A_{4}, A_{b}\right)=0.16072 \\
& D M_{O}\left(A_{5}, A_{b}\right)=0.19381 \\
& D M_{O}\left(A_{6}, A_{b}\right)=0.14857
\end{aligned}
$$

Here, we have ranking

$$
A_{6}>A_{3}>A_{4}>A_{1}>A_{2}>A_{5},
$$

Hence, the most faculty (alternative) is $A_{6}$.

+ Apply the Eq.(10), with the dissimilarity measure $D M_{L}(A, B)$ in the Eq.(8) we have:

$$
\begin{aligned}
& D M_{L}\left(A_{1}, A_{b}\right)=0.21033 \\
& D M_{L}\left(A_{2}, A_{b}\right)=0.22635 \\
& D M_{L}\left(A_{3}, A_{b}\right)=0.18529 \\
& D M_{L}\left(A_{4}, A_{b}\right)=0.19651 \\
& D M_{L}\left(A_{5}, A_{b}\right)=0.23562 \\
& D M_{L}\left(A_{6}, A_{b}\right)=0.18512
\end{aligned}
$$

Here, we have ranking:

$$
A_{6}>A_{3}>A_{4}>A_{1}>A_{2}>A_{5},
$$

where the symbol " > " mean that order relation "superior". Hence, the most faculty (alternative) is $A_{6}$.

+ Apply the Eq.(10), with the dissimilarity measure $D M_{C}(A, B)$ in the Eq.(6) we have:

$$
\begin{aligned}
& D M_{C}\left(A_{1}, A_{b}\right)=0.22877 \\
& D M_{C}\left(A_{2}, A_{b}\right)=0.22411 \\
& D M_{C}\left(A_{3}, A_{b}\right)=0.19316 \\
& D M_{C}\left(A_{4}, A_{b}\right)=0.20167 \\
& D M_{C}\left(A_{5}, A_{b}\right)=0.23985 \\
& D M_{C}\left(A_{6}, A_{b}\right)=0.19934
\end{aligned}
$$

Here, we have ranking: 


$$
A_{3}>A_{6}>A_{4}>A_{2}>A_{1}>A_{5},
$$

hence the most faculty (alternative) is $A_{3}$.

Example 9. Now, we use the distance measure to rank the alternative base on attribute in example 8.

Table 2. Picture Fuzzy Decision Matrix R

\begin{tabular}{|c|c|c|c|}
\hline & $\mathrm{G}_{1}$ & $G_{2}$ & $G_{3}$ \\
\hline $\mathrm{A}_{1}$ & $(0.61,0.08,0.11)$ & $(0.59,0.04,0.26)$ & $(0.41,0.36,0.08)$ \\
\hline $\mathrm{A}_{2}$ & $(0.54,0.45,0.01)$ & $(0.5,0.15,0.25)$ & $(0.66,0.12,0.01)$ \\
\hline $\mathrm{A}_{3}$ & $(0.72,0.23,0.03)$ & $(0.53,0.05,0.35)$ & $(0.5,0.03,0.08)$ \\
\hline $\mathrm{A}_{4}$ & $(0.63,0.3,0.06)$ & $(0.59,0.29,0.08)$ & $(0.58,0.07,0.16)$ \\
\hline $\mathrm{A}_{5}$ & $(0.59,0.3,0.09)$ & $(0.41,0.57,0.01)$ & $(0.59,0.19,0.1)$ \\
\hline $\mathrm{A}_{6}$ & $(0.73,0.1,0.15)$ & $(0.54,0.37,0.06)$ & $(0.46,0.24,0.06)$ \\
\hline
\end{tabular}

The distance measure between alternatives $A_{i}$ and the possible ideal alternative $A_{b}$ is defined as follows:

$$
D\left(A_{i}, A_{b}\right)=\sum_{j=1}^{3} w_{j} D\left(r_{i j}, A_{b}(j)\right)
$$

where $D\left(r_{i j}, A_{b}(j)\right)$ is the distance measure of $r_{i j}$ and $A_{b}(j), j=1,2,3 ; i=1,2, \ldots, 6$.

So that, the smaller of the distance measure is the better alternatives.

We consider the calculated results according to the measurements in the section 3 .

+ Apply the Eq.(11), with the distance measure $D_{H}(A, B)$ in the Eq.(2) we have:

$$
\begin{gathered}
D_{H}\left(A_{1}, A_{b}\right)=0.23417 \\
D_{H}\left(A_{2}, A_{b}\right)=0.275 \\
D_{H}\left(A_{3}, A_{b}\right)=0.21217 \\
D_{H}\left(A_{4}, A_{b}\right)=0.24217 \\
D_{H}\left(A_{5}, A_{b}\right)=0.29083 \\
D_{H}\left(A_{6}, A_{b}\right)=0.2255
\end{gathered}
$$

Here, we have ranking:

$$
A_{3}>A_{6}>A_{1}>A_{4}>A_{2}>A_{5},
$$

where the symbol " $>$ " mean that order relation "superior", hence the most faculty (alternative) is $A_{3}$.

+ Apply the Eq.(11), with the dissimilarity measure $D_{E}(A, B)$ in the Eq.(3) we have: 


$$
\begin{gathered}
D_{E}\left(A_{1}, A_{b}\right)=0.48811 \\
D_{E}\left(A_{2}, A_{b}\right)=0.57081 \\
D_{E}\left(A_{3}, A_{b}\right)=0.44846 \\
D_{E}\left(A_{4}, A_{b}\right)=0.48216 \\
D_{E}\left(A_{5}, A_{b}\right)=0.58144 \\
D_{E}\left(A_{6}, A_{b}\right)=0.4457
\end{gathered}
$$

Here, we have ranking:

$$
A_{6}>A_{3}>A_{4}>A_{1}>A_{2}>A_{5},
$$

Hence, the most faculty (alternative) is $A_{6}$.

+ Apply the Eq.(11), with the distance measure $D_{H}^{m}(A, B)$ in the Eq.(4) we have:

$$
\begin{gathered}
D_{H}^{m}\left(A_{1}, A_{b}\right)=0.435 \\
D_{H}^{m}\left(A_{2}, A_{b}\right)=0.446 \\
D_{H}^{m}\left(A_{3}, A_{b}\right)=0.3715 \\
D_{H}^{m}\left(A_{4}, A_{b}\right)=0.39 \\
D_{H}^{m}\left(A_{5}, A_{b}\right)=0.455 \\
D_{H}^{m}\left(A_{6}, A_{b}\right)=0.3715
\end{gathered}
$$

Here, we have ranking:

$$
A_{3}=A_{6}>A_{4}>A_{1}>A_{2}>A_{5},
$$

Hence, the most faculty (alternative) is $A_{3}, A_{6}$.

This case to see that the distance measure $D_{H}^{m}(A, B)$ is not well as dissimilarity measures, which we use before, be cause It does not know which alternative is better between $A_{3}, A_{6}$.

\section{Conclusion}

In this paper, we introduce the concepts of the difference between PFS-sets, distance measure and dissimilarity between picture fuzzy sets. We give some distant measure and dissimilarity measure of picture fuzzy sets. Beside, we Illustrate with numerical examples the above measures in decision making. In the future, we will study the properties of these measure and applications of them in practical problems. 
Finally, we applied the similarity measures in multiple attribute decision making. Since, we see that dissimilarity is a useful way to deal with realistic problems and can be extended in other application fields.

\section{References}

[1] B. Bouchon-Meunier, M. Rifqi, and S. Bothorel. "Towards general measures of comparison of objects". Fuzzy sets and systems, 84(2), pp 143-153, 1996.

[2] B. C., Cuong, "Picture Fuzzy Sets". Journal of Computer Science and Cybernetics 30(4), p 409-420, 2014.

[3] B. C. Cuong and P. Van Hai., October). "Some fuzzy logic operators for picture fuzzy sets". In Knowledge and Systems Engineering (KSE), 2015 Seventh International Conference on , pp. 132-137. IEEE, 2015.

[4] P. A. Ejegwa, A. J. Akubo, and O. M Joshua. "Intuitionistic fuzzy set and its application in career determination via normalized Euclidean distance method". European Scientific Journal, ESJ, 10(15), 2014

[5] F. Faghihi. "Generalization of the dissimilarity measure of fuzzy sets". In International Mathematical Forum . Vol. 2, No. 68, pp. 3395-3400, 2007.

[6] A. G. Hatzimichailidis, G. A. Papakostas, and V. G. Kaburlasos. "A novel distance measure of intuitionistic fuzzy sets and its application to pattern recognition problems". International Journal of Intelligent Systems, 27(4), pp 396-409, 2012.

[7] D. F. Li. "Some measures of dissimilarity in intuitionistic fuzzy structures". Journal of Computer and System Sciences, 68(1), pp 115-122, 2004.

[8] J. Lindblad and N. Sladoje. "Linear time distances between fuzzy sets with applications to pattern matching and classification". IEEE Transactions on Image Processing, 23(1), pp 126-136,2014 .

[9] S. Maheshwari and A. Srivastava. "Study on divergence measures for intuitionistic fuzzy sets and its application in medical diagnosis". Journal of Applied Analysis and Computation, 6(3), pp 772-789, 2016.

[10] S. Mahmood. "Dissimilarity Fuzzy Soft Points and Their Applications". Fuzzy Information and Engineering, 8(3), pp 281-294, 2016.

[11] I. Montes, N. R. Pal., V. Janiš and S. Montes. "Divergence measures for intuitionistic fuzzy sets". IEEE Transactions on Fuzzy Systems, 23(2), pp. 444-456, 2015.

[12] P. Muthukumar and G. S. S. Krishnan. "A similarity measure of intuitionistic fuzzy soft sets and its application in medical diagnosis". Applied Soft Computing, 41, pp 148-156, 2016.

[13] H. Nguyen. "A novel similarity/dissimilarity measure for intuitionistic fuzzy sets and its application in pattern recognition". Expert Systems with Applications, 45, pp 97-107, 2016.

[14] H. D. Nguyen, S. H. Le, and T.H. Pham. "Some Improvements of Fuzzy Clustering Algorithms Using Picture Fuzzy Sets and Applications For Geographic Data Clustering”. VNU Journal of Science: Computer Science and Communication Engineering, 32(3), 2016.

[15] X.T. Nguyen, L.H. Son, B. C. Cuong, "Mumtaz Ali, Luong Hong Lan, Fuzzy equivalence on standard and rough neutrosophic sets and applications to clustering analysis", Proceeding of the 2017 4th International Conference on Information System Design and Intelligent Applications (INDIA-2017), 1517 June 2017, Danang, Vietnam, Springer (In press).

[16] L. H. Son. “A Novel Kernel Fuzzy Clustering Algorithm for Geo-Demographic Analysis”. Information Sciences 317, pp $202-223,2015$.

[17] L. H. Son (2016), Generalized Picture Distance Measure and Applications to Picture Fuzzy Clustering. Applied Soft Computing 46, pp 284-295, 2016.

[18] L. H. Son, P. V. Viet, and P.V Hai. "Picture Inference System: A New Fuzzy Inference System on Picture Fuzzy Set”. Applied Intelligence, 2017. DOI: 10.1007/s10489-016-0856-1. 
[19] N. Van Dinh, N. X. Thao and N.M. Chau. "On the picture fuzzy database: theories and application", $J$. Sci, 13(6), 1028-1035, 2015.

[20] Z. S. Xu. "Models for multiple attribute decision making with intuitionistic fuzzy information". International Journal of Uncertainty, Fuzziness and Knowledge-Based Systems, 15(03), 285-297, 2007.

\section{Authors' Profiles}

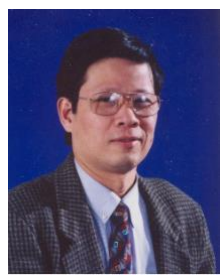

V.D. Nguyen was born on the year 1953 in Hanoi, Vietnam. He was a post-graduate intern in computer Science in Moscow, The Russian Federation. In 2004 he received the Doctor of Philosophy (PhD) Degree in Mathematics at the College of Science, Vietnam National University (VNU), Hanoi, Vietnam. Now he is an Associate Professor in the Department of applied Math-Informatics, Faculty of Information Technology, Vietnam National University of Agriculture (VNUA), Hanoi, Vietnam. He also is a member of the Editorial Board for the International Journal of Intelligent Systems and Applications, MECS Publisher.

Some articales on similarity topics he has published: Rough Fuzzy Relation on Two Universal Sets, I.J. Intelligent Systems and Applications, 2014, 04, 49-55; "Support-intuitionistic fuzzy set: a new concept for soft computing". International Journal of Intelligent Systems and Applications, ISSN: 2074-904X. MECS Press, Hong Kong. 2015, 04. p. 11 - 16; "Support-Neutrosophic Set: A New Concept in Soft Computing", International Journal in Neutrosophic Sets and Systems, New Mexico University Press, NM 87301, USA. ISSN 2331-6055. Vol. 16, 2017, p.93-98.

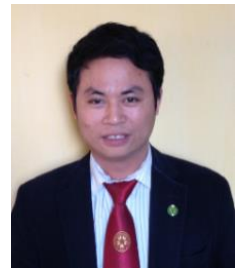

X.T. Nguyen was born on October 28, 1982, in Thai Binh, Viet Nam. He received the B.Sc. and M.S Degrees, in Mathematic from The College of Science Vietnamese National University (VNU), Hanoi, in 2004 and 2009, respectively. Now, he is a lecturer, Department of applied Math-Informatics, Faculty of Information Technology, Vienam National University of Agriculture (VNUA), Viet Nam. He is teaching Calculus, Optimization, Fuzzy logic and its application. His research interests are Spectral theory of operator Fuzzy set theory, Rough set theory and application in data mining. Some articales on similarity topics he has published: Remarks on the spectrum of a compact convex set of compact operators, Journal of Applied Analysis 16 (2010), pp 259-264; Rough Fuzzy Relation on Two Universal Sets, I.J. Intelligent Systems and Applications, 2014, 04, 49-55; "Support-intuitionistic fuzzy set: a new concept for soft computing”. International Journal of Intelligent Systems and Applications, ISSN: 2074-904X. MECS Press, Hong Kong. 2015, 04. p. 11 - 16; "Support-Neutrosophic Set: A New Concept in Soft Computing", International Journal in Neutrosophic Sets and Systems, New Mexico University Press, NM 87301, USA. ISSN 2331-6055. Vol. 16, 2017, p.93-98.

How to cite this paper: Nguyen Van Dinh, Nguyen Xuan Thao,"Some Measures of Picture Fuzzy Sets and Their Application in Multi-attribute Decision Making", International Journal of Mathematical Sciences and Computing(IJMSC), Vol.4, No.3, pp.23-41, 2018.DOI: 10.5815/ijmsc.2018.03.03 